\title{
The aqueous-phase synthesis of sulfonylthioureas and a study of their properties as anion receptors
}

\author{
Cong-Wen Ding, * Hai-Feng Yu, ${ }^{\text {a }}$ Rui-Min Li, ${ }^{\mathbf{b}}$ Xiang-Fu Jiang, a and Bin Luo \\ ${ }^{a}$ Institute of Fine Chemical Industry, Department of Chemistry and Chemical Indutry, College of \\ Life Sciences, Tarim University, Alaer843300, Xinjiang, People's Republic of China \\ ${ }^{b}$ Tianjin Pharmaceutical Tech-Development Co.Ltd., Tianjin300193, People's Republic of China \\ E-mail: dingcw19731008@163.com
}

\begin{abstract}
A series of mono- or bis-sulfonylthioureas has been synthesized via nucleophilic substitution of potassium sulfonamides and sodium dithiocarbamate in water under mild conditions, and their properties as anion receptors in the absence and presence of $\mathrm{K}^{+}$have been investigated by ${ }^{1} \mathrm{H}$ NMR spectroscopy. It was found that calix[4]crown-5 derivative $\mathbf{1 0}$ shows much higher selective complexation capability with $\mathrm{H}_{2} \mathrm{PO}_{4}{ }^{-}$in the presence of $\mathrm{K}^{+}$than in the absence of $\mathrm{K}^{+}$, which indicates the physical dimension of $\mathrm{K}^{+}$and $\mathrm{H}_{2} \mathrm{PO}_{4}{ }^{-}$, respectively, matched the corresponding cavity of the host compound.
\end{abstract}

Keywords: Synthesis, sulfonylthiourea, sulfonamide, dithiocarbamate, anion receptor, water

\section{Introduction}

Thioureas are useful reagents for f. ex. anion complexation ${ }^{1}$ and sensing ${ }^{2}$, asymmetric catalysts ${ }^{3}$ and solar cell construction ${ }^{4}$. The derived sulfonylthioureas display a broad spectrum of biological and pharmacological properties with action as antidiabetics ${ }^{5}$, enhancers of parasympathetic system function ${ }^{6}$, anticonvulsants ${ }^{7}$, antiarrhythmics ${ }^{8}$ or herbicides ${ }^{9}$. Furthermore, they are often used as synthetic intermediates ${ }^{10}$ for a variety of guanidine drugs and also as building blocks ${ }^{11}$ in the synthesis of heterocycles. Direct condensation of sulfonylisothiocyanate with amine in dry $\mathrm{CHCl}_{3}$ or $\mathrm{CH}_{2} \mathrm{Cl}_{2}$ is the general procedure for the synthesis of sulfonylthioureas. ${ }^{12}$ Another synthetic route ${ }^{13}$ is nucleophilic addition of sulfonamide and isothiocyanate in the presence of inorganic bases in anhydrous acetone, DMF or DMSO. The two methods are frequently limited by harsh reaction conditions, lower yields, longer reaction times, and use of toxic solvents. To the best of our knowledge, the voluminous literature about applications of sulfonylthioureas does not mention the use of sulfonylthioureas as anion receptors probably due to their inaccessibility by traditional synthetic methods. We now report the aqueous-phase synthesis of sulfonylthioureas and a study of their properties as anion receptors. 


\section{Results and Discussion}

\section{Aqueous-phase synthesis of sulfonylthioureas}

The aqueous-phase synthetic method we offered holds many advantages such as mild reaction conditions, free organic solvent, high isolated product yields and very short reaction time. Thus it provided a better and more practical alternative to existing procedures. The synthetic route is outlined in Scheme 1. $\mathrm{C}=\mathrm{S}$ of $\mathbf{1 2}$ has been activated by $\mathrm{ClCH}_{2} \mathrm{COONa}$. The reaction time is shorter for the preparation of $\mathbf{7}$ and 10. It is because that benzo-18-crown-6 and calix[4]crown-5 can effectively bind $\mathrm{K}^{+}$from 11. $\mathrm{ArSO}_{2} \mathrm{NH}^{-}$become unsheltered, which makes nucleophilicity of 11 strengthened.
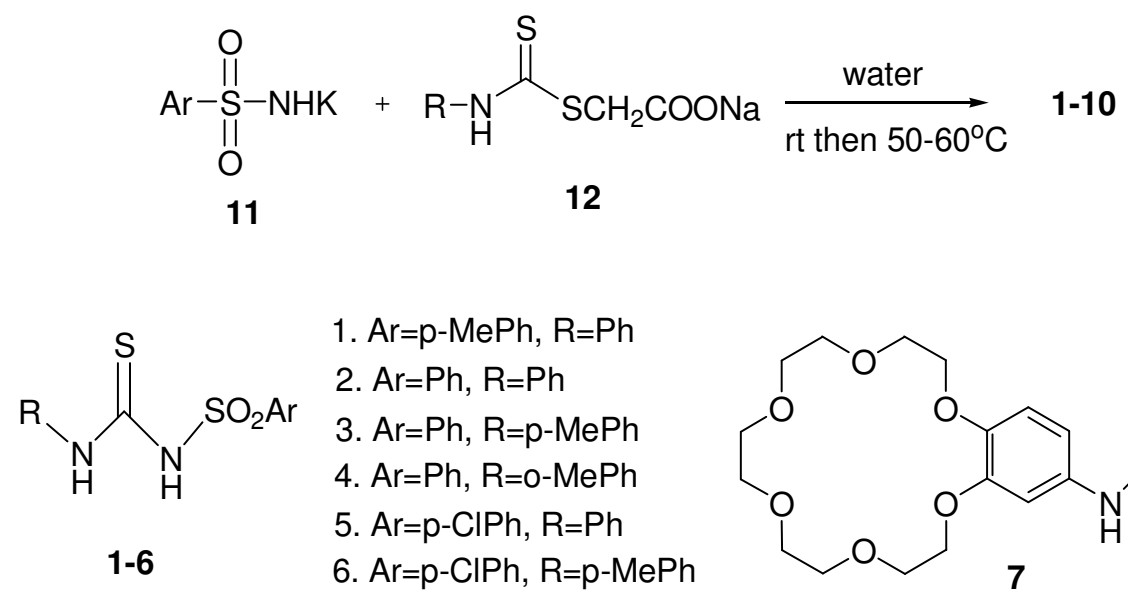<smiles>[3H]CCOc1cc(NC(=S)NS(=O)(=O)c2ccccc2)ccc1OCCOCCOCCOCCO</smiles>

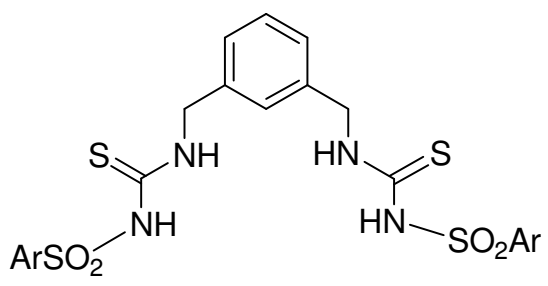

8-9

8. $\mathrm{Ar}=\mathrm{Ph}$

9. Ar=1-naphthyl

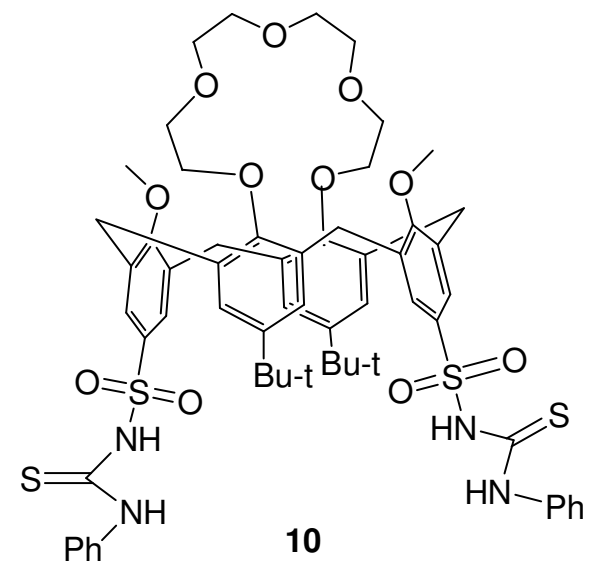

Scheme 1. Aqueous-phase synthesis of derived sulfonylthioures 1-10 under mild conditions.

Sulfonylthioureas 1-10 has been synthesized via nucleophilic substitution of potassium sulfonamides 11 and dithiocarbamate 12 at rt to $50-60{ }^{\circ} \mathrm{C}$ within $1.0 \mathrm{~h}$ in water. Aqua 11 were prepared from $\mathrm{ArSO}_{2} \mathrm{NH}_{2}$ and equivalent $\mathrm{KOH}$ in water. Corresponding $\mathrm{ArSO}_{2} \mathrm{NH}_{2}$ for the synthesis of compound $\mathbf{1 0}$ was obtained based on the modified procedures. ${ }^{14 a, 15}$ Aqua $\mathbf{1 2}$ were afforded from sodium dithiocarbamate and $\mathrm{ClCH}_{2} \mathrm{COONa}$ in water following the method. ${ }^{14 \mathrm{~b}}$ All 
new compounds were characterized by IR, ${ }^{1} \mathrm{H}$ NMR, ${ }^{13} \mathrm{C}$ NMR, mass spectra and elemental analysis.

In conclusion, we have designed and developed a convenient, efficient, economically and environmentally benign procedure for the synthesis of sulfonylthioureas which will be studied below for their properties as anion receptors.

\section{Sulfonylthiourea-based properties as anion receptors.}

$\mathbf{2}$ and 7-10 were selected as test objects. Their properties as anion receptors were investigated by standard ${ }^{1} \mathrm{H}$ NMR titration using a ligands concentration (0.1-2.0 mM) and an increasing concentration of appropriate anion to obtain different host : guest ratios $(0.1-20: 1)$. To ensure the solubility of both organic ligands and inorganic anions, a mixed solvent system $\left(\mathrm{CDCl}_{3}{ }^{-}\right.$ $\mathrm{CD}_{3} \mathrm{CN} 4: 1$, v/v) was used. All anions were added as their tetrabutylammonium salts to minimize possible interactions of sulfonylthioureas with counter cations. The addition of anions led to the down-field shifts of $-\mathrm{SO}_{2} \mathrm{NH}^{-}$signals. Another experiment was carried out repeatedly in the presence of 2 eq. of $\mathrm{KBPh}_{4}$ by the same procedure as described above.

Results are summarized in Table 1 and 2 where diphenylthiourea is taken as a reference. The binding constants of $\mathbf{8}$ and $\mathbf{1 0}$ towards some anions are too large to be determined accurately by ${ }^{1} \mathrm{H}$ NMR spectroscopy and the values given are rough estimations.

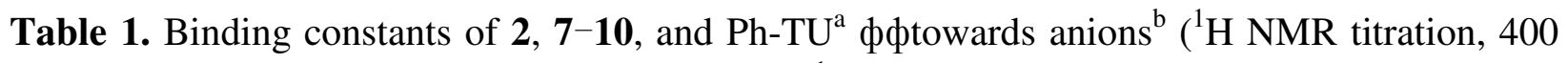
$\mathrm{MHz}, \mathrm{CDCl}_{3}-\mathrm{CD}_{3} \mathrm{CN}=4: 1, \mathrm{v} / \mathrm{v}, 25^{\circ} \mathrm{C}, \mathrm{Kass}, \mathrm{M}^{-1}$ )

\begin{tabular}{ccccccc}
\hline anion $^{\text {b }}$ & Ph-TU & Receptor 2 & Receptor 7 & Receptor 8 & Receptor 9 & $\begin{array}{c}\text { Receptor } \\
\mathbf{1 0}\end{array}$ \\
\hline $\mathrm{H}_{2} \mathrm{PO}_{4}{ }^{-}$ & 420 & 740 & 720 & 51000 & 1600 & 350000 \\
$\mathrm{HSO}_{4}{ }^{-}$ & 170 & 260 & 220 & 1300 & 350 & 1470 \\
$\mathrm{I}^{-}$ & 6 & 8 & 11 & 9 & 5 & 165 \\
$\mathrm{Br}^{-}$ & 10 & 17 & 15 & 14 & 16 & 2000 \\
$\mathrm{Cl}^{-}$ & 13 & 26 & 19 & 18 & 25 & 2500 \\
\hline
\end{tabular}

${ }^{\mathrm{a}} \mathrm{Ph}-\mathrm{TU}$ : diphenylthiourea. ${ }^{\mathrm{b}}$ Counter-cation: $\mathrm{NBu}_{4}{ }^{+}$.

Table 1 shows that the association constants follow the trend $\mathrm{H}_{2} \mathrm{PO}_{4}{ }^{-}>\mathrm{HSO}_{4}{ }^{-}>\mathrm{Cl}^{-}>$ $\mathrm{Br}^{-}>\mathrm{I}^{-}$for $\mathbf{2}$ and $\mathbf{7 - 1 0}$ as well as Ph-TU. The observed binding selectivity can be roughly explained by the anion basicity order. $\mathbf{2}$ and 7-10 lead to much higher complexation constants if compared with the reference. This can be understood by considering the electron-withdrawing effect of $\mathrm{SO}_{2}$ groups. The influence of the naphthyl group of $\mathbf{9}$ is less beneficial for complexation likely because of a decrease in electron delocalization energy. 10 showed higher selective affinity for $\mathrm{H}_{2} \mathrm{PO}_{4}{ }^{-}$due to the enhanced hydrogen bonding ability of the sulfonylthiourea ${ }^{-} \mathrm{NH}$ protons. Most important of all, the cavity of $\mathbf{1 0}$ matched the size and shape of given $\mathrm{H}_{2} \mathrm{PO}_{4}{ }^{-}$. 
Table 2 indicates that $\mathbf{7}$ and $\mathbf{1 0}$ work as a novel bifunctional receptor for simultaneous complexation of $\mathrm{K}^{+}$and anions, where the ability of $\mathbf{7}$ and $\mathbf{1 0}$ to bind anions is significantly enhanced when $\mathrm{K}^{+}$is bound to the crown moiety. This is supported by their ${ }^{1} \mathrm{H}$ NMR spectroscopy, in which downfield shifts for sulfonylthiourea protons were observed upon complexation of $\mathrm{K}^{+}$at the crown moiety. But, no complexes of crown moiety can be detected when $\mathrm{K}^{+}$is replaced by the other metal ion such as $\mathrm{Na}^{+}, \mathrm{Rb}^{+}$, and $\mathrm{Cs}^{+}$. By contrast, $\mathrm{Ph}-\mathrm{TU}, \mathbf{2}, \mathbf{8}$, and $\mathbf{9}$, which lack crown ether moiety for $\mathrm{K}^{+}$binding, shows a small decrease for anions. $\mathbf{1 0}$ can act as a better bifunctional receptor. Selective complexation model of 10 with $\mathrm{H}_{2} \mathrm{PO}_{4}{ }^{-}$in the presence of $\mathrm{K}^{+}$is depicted in Scheme 2 .

Table 2. Binding constants of $\mathbf{2 , 7 - 1 0}$, and $\mathrm{Ph}-\mathrm{TU}^{\mathrm{a}}$ towards anions ${ }^{\mathrm{b}}\left({ }^{1} \mathrm{H}\right.$ NMR titration, $400 \mathrm{MHz}$, $\mathrm{CDCl}_{3}-\mathrm{CD}_{3} \mathrm{CN} 4: 1, \mathrm{v} / \mathrm{v}, 25^{\circ} \mathrm{C}$, Kass, $\mathrm{M}^{-1}$ ) in the presence of (2eq.) $\mathrm{K}^{+\mathrm{c}}$

\begin{tabular}{ccccccc}
\hline Anion $^{\text {b }}$ & Ph-TU & Receptor 2 & Receptor 7 & Receptor 8 & Receptor 9 & $\begin{array}{c}\text { Receptor } \\
\mathbf{1 0}\end{array}$ \\
\hline $\mathrm{H}_{2} \mathrm{PO}_{4}^{-}$ & 418 & 735 & 7500 & 50000 & 1590 & 3700000 \\
$\mathrm{HSO}_{4}^{-}$ & 165 & 255 & 600 & 1250 & 340 & 9470 \\
$\mathrm{I}^{-}$ & 5 & 4 & 110 & 7 & 4 & 1650 \\
$\mathrm{Br}^{-}$ & 9 & 16 & 150 & 12 & 14 & 2100 \\
$\mathrm{Cl}^{-}$ & 11 & 23 & 190 & 15 & 22 & 2800 \\
\hline
\end{tabular}

${ }^{\mathrm{a}} \mathrm{Ph}$-TU: diphenylthiourea. ${ }^{\mathrm{b}}$ Counter-cation: $\mathrm{NBu}_{4}{ }^{+} \cdot{ }^{\mathrm{c}}$ Counter-anion: $\mathrm{BPh}_{4}{ }^{-}$

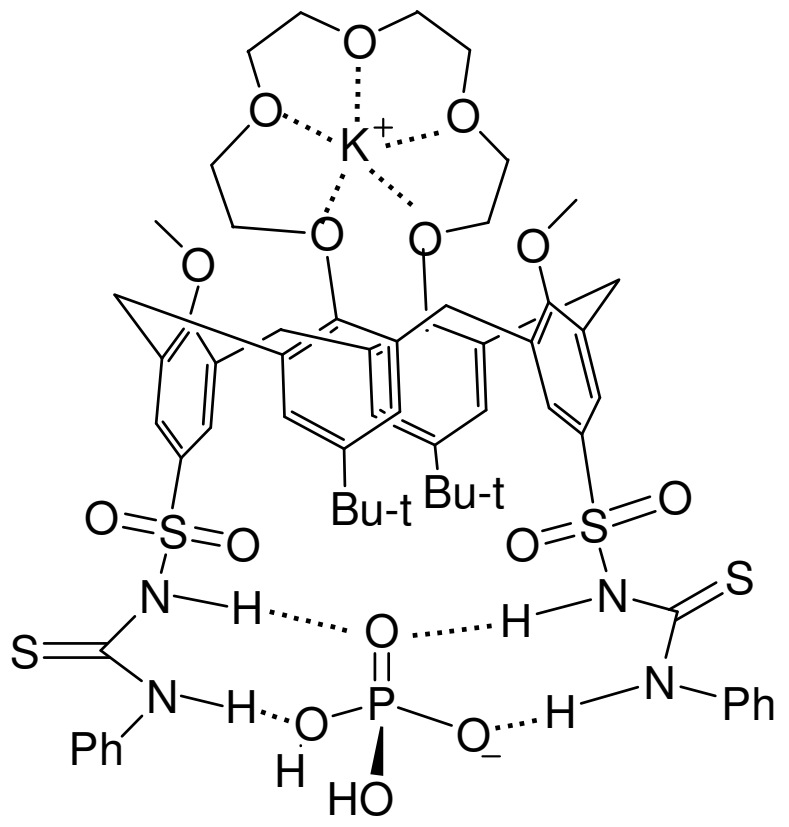

Scheme 2. Possible complexation model of $\mathbf{1 0}$ with $\mathrm{H}_{2} \mathrm{PO}_{4}{ }^{-}$in the presence of $\mathrm{K}^{+}$ 


\section{Conclusions}

In this study, a series of mono- or bis-sulfonylthioureas as potential hydrogen bond donors to bind some anions, has been synthesized via nucleophilic substitution of potassium sulfonamides and sodium dithiocarbamate in water under mild conditions, and the anion complexation in the absence and presence of $\mathrm{K}^{+}$has been examined by ${ }^{1} \mathrm{H}$ NMR spectroscopy. The presence of electron withdrawing sulfonyl group results in very high binding affinity towards anions compared with reference Ph-TU. $\mathbf{7}$ and $\mathbf{1 0}$ bearing crown ether moiety are found to work as a novel bifunctional receptor for simultaneously selective complexation of $\mathrm{K}^{+}$and $\mathrm{H}_{2} \mathrm{PO}_{4}{ }^{-}$, where the ability of $\mathbf{7}$ and $\mathbf{1 0}$ to bind $\mathrm{H}_{2} \mathrm{PO}_{4}{ }^{-}$is significantly enhanced when $\mathrm{K}^{+}$is bound to the crown moiety, whereas receptor 10 shows a much higher complexation degree with $\mathrm{H}_{2} \mathrm{PO}_{4}{ }^{-}$due to the cavities of crown moiety and calixarene matching the physical dimensions of $\mathrm{K}^{+}$and $\mathrm{H}_{2} \mathrm{PO}_{4}{ }^{-}$, respectively.

\section{Experimental Section}

General. The reagents used were of reagent grade and used as purchased. Melting points were measured by using a XT-4A Electrothermal micro-melting-point apparatus and are uncorrected. NMR spectra were recorded at $400\left({ }^{1} \mathrm{H}\right)$ and $100\left({ }^{13} \mathrm{C}\right) \mathrm{MHz}$, respectively, on a Bruker Avance III Plus 400 spectrometer in DMSO- $\mathrm{d}_{6}$ using TMS as internal reference. Infrared spectra were obtained on a Bio-Rad spectrophotometer using $\mathrm{KBr}$ pellets. Mass spectra were measured using Agilent 6100B and Agilent 6220 Time-of-Flight LC/MS. Elemental analysis was determined on a Vario EL III elemental analyzer.

Typical procedure to synthesize sulfonylthioureas (1-10). To the aqueous solution of $\mathrm{ArSO}_{2} \mathrm{NHK} 11$ (1 eq.) was dropwise added an aqueous solution of sodium dithiocarbamates 12 (1eq.). After the addition was completed, the reaction mixture was stirred for about $30 \mathrm{~min}$ at room temperature and was subsequently heated to $50-60{ }^{\circ} \mathrm{C}$ for another $30 \mathrm{~min}$ or so. Finally, the mixture was cooled to room temperature.The resulting precipitate was collected by suction filtration and washed successively with $\mathrm{H}_{2} \mathrm{O}$, then dried under vacuum over $\mathrm{P}_{4} \mathrm{O}_{10}$ to afford sulfonylthioureas 1-10 in high purity and yields.

Sulfonylthiourea 1.Yield, 85\%, white crystalline powder, mp 146-148 ${ }^{\circ} \mathrm{C}$ (lit. ${ }^{16}$ : $144-146{ }^{\circ} \mathrm{C}$ ); ${ }^{1} \mathrm{H}$ NMR (DMSO- $\left.d_{6}, 400 \mathrm{MHz}\right) \delta(\mathrm{ppm}): 9.75$ (brs, $\left.1 \mathrm{H}\right), 8.41$ (brs, $\left.1 \mathrm{H}\right), 7.82(\mathrm{~d}, J=8.3 \mathrm{~Hz}, 2 \mathrm{H}$ ), 7.47-7.26 (m, 7H), 2.46 (s, 3H). IR (KBr, $\left.\mathrm{cm}^{-1}\right)$ v: 3302, 1597, 1518, 1481, 1381, 1178, 1146, $1082 \mathrm{~cm}^{-1}$. HRMS (ESI) calcd for $\mathrm{C}_{14} \mathrm{H}_{15} \mathrm{~N}_{2} \mathrm{O}_{2} \mathrm{~S}_{2}\left(\mathrm{M}+\mathrm{H}^{+}\right)$307.0575, found 307.0566.

Sulfonylthiourea 2. Yield, 82\%, white crystalline powder, mp 135-137 ${ }^{\circ} \mathrm{C}$ (lit. ${ }^{17}: 120{ }^{\circ} \mathrm{C}$ ). ${ }^{1} \mathrm{H}$ NMR (DMSO- $\left.d_{6}, 400 \mathrm{MHz}\right) \delta(\mathrm{ppm}): 9.76$ (brs, $\left.1 \mathrm{H}\right), 8.80$ (brs, $\left.1 \mathrm{H}\right), 7.96$ (d, $J=7.5 \mathrm{~Hz}, 2 \mathrm{H}$ ), 7.74-7.29 (m, 8H). IR (KBr, cm $\left.{ }^{-1}\right)$ v: 3285, 1593, 1537, 1481, 1447, 1383, $1084 \mathrm{~cm}^{-1}$. ESI-MS 
$m / z: 293.2\left(\mathrm{M}+\mathrm{H}^{+}\right)$. Anal. calcd for $\mathrm{C}_{13} \mathrm{H}_{12} \mathrm{~N}_{2} \mathrm{O}_{2} \mathrm{~S}_{2}$ : C 53.40, H 4.14, N 9.58; found C 53.14, $\mathrm{H}$ 4.35, N 9.70 .

Sulfonylthiourea 3. Yield, 82\%, white crystalline powder, mp 149-151 ${ }^{\circ} \mathrm{C}$ (lit. ${ }^{17}: 124{ }^{\circ} \mathrm{C}$ ). ${ }^{1} \mathrm{H}$ NMR $\left(400 \mathrm{MHz}, \mathrm{DMSO}-d_{6}\right) \delta$ (ppm): 9.68 (brs, $\left.1 \mathrm{H}\right), 8.55$ (brs, $\left.1 \mathrm{H}\right), 7.95(\mathrm{~d}, J=7.3 \mathrm{~Hz}, 2 \mathrm{H})$, 7.71-7.57 (m, 3H), 7.28 (d, $J=8.4 \mathrm{~Hz}, 2 \mathrm{H}), 7.19$ (d, $J=8.4 \mathrm{~Hz}, 2 \mathrm{H}), 2.35$ (s, 3H). IR (KBr, cm $\left.{ }^{-1}\right)$ v: $3298,1595,1533,1474,1448,1385,1337,1186,1142,1084 \mathrm{~cm}^{-1}$. ESI-MS m/z: 307.1(M+ $\mathrm{H}^{+}$). Anal. calcd for $\mathrm{C}_{14} \mathrm{H}_{14} \mathrm{~N}_{2} \mathrm{O}_{2} \mathrm{~S}_{2}$ : C 54.88, H 4.61, N 9.14; found C 54.58, H 4.99, N 9.14.

Sulfonylthiourea 4. Yield, $82 \%$, white crystalline powder, mp $168-170{ }^{\circ} \mathrm{C} .{ }^{1} \mathrm{H}$ NMR $(400 \mathrm{MHz}$, DMSO- $\left.d_{6}\right) \delta(\mathrm{ppm}): 9.47$ (brs, $\left.1 \mathrm{H}\right), 8.80$ (brs, $\left.1 \mathrm{H}\right), 7.99(\mathrm{~d}, J=7.5 \mathrm{~Hz}, 2 \mathrm{H}), 7.76-7.58(\mathrm{~m}, 3 \mathrm{H})$, 7.29-7.20 (m, 4H), $2.09(\mathrm{~s}, 3 \mathrm{H}) .{ }^{13} \mathrm{C}$ NMR $\left(100 \mathrm{MHz}, \mathrm{DMSO}-d_{6}\right) \delta(\mathrm{ppm}): 177.8,138.4$, $135.8,134.5,134.3,131.0,129.7,128.3,127.2,126.9,126.8,17.7 . \mathrm{IR}\left(\mathrm{KBr}, \mathrm{cm}^{-1}\right)$ v: 3316,3288 , $1510,1476,1447,1375,1186,1167,1144,1084 \mathrm{~cm}^{-1}$. ESI-MS $\mathrm{m} / z: 307.1\left(\mathrm{M}+\mathrm{H}^{+}\right)$. Anal. calcd for $\mathrm{C}_{14} \mathrm{H}_{14} \mathrm{~N}_{2} \mathrm{O}_{2} \mathrm{~S}_{2}$ : C 54.88, H 4.61, N 9.14; found C 54.49, H 5.00, N 9.13.

Sulfonylthiourea 5. Yield, 84\%, white crystalline powder, mp $138-140{ }^{\circ} \mathrm{C} .{ }^{1} \mathrm{H}$ NMR $(400 \mathrm{MHz}$, DMSO- $\left.d_{6}\right) \delta(\mathrm{ppm}): 9.70$ (brs, $\left.1 \mathrm{H}\right), 8.72$ (brs, $\left.1 \mathrm{H}\right), 7.89$ (d, $\left.J=8.6 \mathrm{~Hz}, 2 \mathrm{H}\right), 7.56$ (d, $J=8.6 \mathrm{~Hz}$, $2 \mathrm{H}), 7.51-7.27(\mathrm{~m}, 5 \mathrm{H}) .{ }^{13} \mathrm{C}$ NMR (100 MHz, DMSO- $\left.d_{6}\right) \delta$ (ppm): 176.6, 141.3, 137.0, 136.7, 130.1, 129.2, 128.6, 127.5, 124.4. IR $\left(\mathrm{KBr}, \mathrm{cm}^{-1}\right)$ v: 3304, 1574, 1516, 1475, 1454, 1385, 1186, $1172,1146,1082 \mathrm{~cm}^{-1}$. ESI-MS $m / z: 327.0\left(\mathrm{M}+\mathrm{H}^{+}\right)$. Anal. calcd for $\mathrm{C}_{13} \mathrm{H}_{11} \mathrm{ClN}_{2} \mathrm{O}_{2} \mathrm{~S}_{2}: \mathrm{C} 47.78$, H 3.39, N 8.57; found C 47.62, H 3.54, N 8.38.

Sulfonylthiourea 6. Yield, $84 \%$, white crystalline powder, mp 174-176 ${ }^{\circ} \mathrm{C} .{ }^{1} \mathrm{H}$ NMR $(400 \mathrm{MHz}$, DMSO- $d_{6}$ ) $\delta$ (ppm): 9.63 (brs, $\left.1 \mathrm{H}\right), 8.49$ (brs, $\left.1 \mathrm{H}\right), 7.88(\mathrm{~d}, J=8.7 \mathrm{~Hz}, 2 \mathrm{H}), 7.56(\mathrm{~d}, J=8.7 \mathrm{~Hz}$, 2H), $7.29(\mathrm{~d}, J=8.3 \mathrm{~Hz}, 2 \mathrm{H}), 7.20(\mathrm{~d}, J=8.3 \mathrm{~Hz}, 2 \mathrm{H}), 2.36(\mathrm{~s}, 3 \mathrm{H}) .{ }^{13} \mathrm{C}$ NMR $(100 \mathrm{MHz}$, DMSO- $\left.d_{6}\right) \delta(\mathrm{ppm}): 176.6,141.3,137.6,136.7,134.5,130.1,129.8,128.6,124.4,21.1 . \mathrm{IR}(\mathrm{KBr}$, $\left.\mathrm{cm}^{-1}\right)$ v: $3310,1578,1522,1475,1387,1178,1146,1080 \mathrm{~cm}^{-1}$. ESI-MS $m / z: 341.0\left(\mathrm{M}+\mathrm{H}^{+}\right)$. Anal. calcd for $\mathrm{C}_{14} \mathrm{H}_{13} \mathrm{ClN}_{2} \mathrm{O}_{2} \mathrm{~S}_{2}$ : C 49.33, H 3.84, N 8.22; found C 49.33, H 3.69, N 8.12.

Sulfonylthiourea 7. Yield, $81 \%$, white crystalline powder, mp 104-106 ${ }^{\circ} \mathrm{C} .{ }^{1} \mathrm{H}$ NMR $(400 \mathrm{MHz}$, DMSO- $\left.d_{6}\right) \delta(\mathrm{ppm}) .{ }^{1} \mathrm{H}$ NMR (400 MHz, DMSO- $\left.d_{6}\right) \delta(\mathrm{ppm}): 9.61$ (brs, $\left.1 \mathrm{H}\right), 8.46$ (brs, $\left.1 \mathrm{H}\right)$, $7.95(\mathrm{~d}, J=7.8 \mathrm{~Hz}, 1 \mathrm{H}), 7.70-7.25(\mathrm{~m}, 4 \mathrm{H}), 7.17(\mathrm{~d}, J=8.9 \mathrm{~Hz}, 1 \mathrm{H}), 7.06(\mathrm{~d}, J=8.9 \mathrm{~Hz}, 1 \mathrm{H})$, $6.89(\mathrm{~d}, J=5.8 \mathrm{~Hz}, 1 \mathrm{H}), 4.25(\mathrm{~m}, 4 \mathrm{H}), 4.21(\mathrm{~m}, 8 \mathrm{H}), 4.11(\mathrm{~m}, 8 \mathrm{H}) .{ }^{13} \mathrm{C}$ NMR $(100 \mathrm{MHz}$, DMSO- $\left.d_{6}\right) \delta(\mathrm{ppm}): 179.6,147.0,142.6,139.7,132.5,129.3,129.1,127.3,118.7,115.3,110.4$, 71.2, 70.6, 70.5, 69.4, 69.3. IR $\left(\mathrm{KBr}, \mathrm{cm}^{-1}\right)$ v: $3280,3220,1576,1520,1477,1389,1176,1144$, $1082 \mathrm{~cm}^{-1}$. ESI-MS m/z: $527.14\left(\mathrm{M}+\mathrm{H}^{+}\right)$. Anal. calcd for $\mathrm{C}_{23} \mathrm{H}_{30} \mathrm{~N}_{2} \mathrm{O}_{8} \mathrm{~S}_{2}: \mathrm{C} 52.46, \mathrm{H} 5.74, \mathrm{~N}$ 5.32; found C 52.43, H 5.79, N 5.29.

Sulfonylthiourea 8. Yield, 79\%, white crystalline powder, mp $184-186{ }^{\circ} \mathrm{C} .{ }^{1} \mathrm{H}$ NMR $(400 \mathrm{MHz}$, DMSO- $\left.d_{6}\right) \delta(\mathrm{ppm}):{ }^{1} \mathrm{H}$ NMR (400 MHz, DMSO- $\left.d_{6}\right) \delta(\mathrm{ppm}): 9.80$ (brs, $\left.2 \mathrm{H}\right), 8.82$ (brs, $2 \mathrm{H}$ ), $7.98(\mathrm{~d}, J=8.1 \mathrm{~Hz}, 2 \mathrm{H}), 7.93-7.54(\mathrm{~m}, 8 \mathrm{H}), 7.02(\mathrm{dd}, J=7.3 \mathrm{~Hz}, 1 \mathrm{H}), 6.87(\mathrm{~d}, J=7.3 \mathrm{~Hz}, 2 \mathrm{H})$, $6.86(\mathrm{~d}, J=5.3 \mathrm{~Hz}, 1 \mathrm{H}), 4.79(\mathrm{~d}, J=5.1 \mathrm{~Hz}, 4 \mathrm{H}) .{ }^{13} \mathrm{C}$ NMR $\left(100 \mathrm{MHz}\right.$, DMSO- $\left.d_{6}\right) \delta(\mathrm{ppm})$ : 184.1, 141.5, 139.7, 132.0, 129.1, 128.4, 127.3, 126.7, 125.4, 51.3. IR (KBr, cm $\left.{ }^{-1}\right)$ v: 3376, 3276, 3162, 3059, 2933, 2869, 1579, 1385, 1168, 1149, 1080, 707, $694 \mathrm{~cm}^{-1}$. ESI-MS m/z: $535.05(\mathrm{M}$ $+\mathrm{H}^{+}$). Anal. calcd for $\mathrm{C}_{22} \mathrm{H}_{22} \mathrm{~N}_{4} \mathrm{O}_{4} \mathrm{~S}_{4}$ : C 49.42, H 4.15, N 10.48; found C 49.41, H 4.19, N 10.45. 
Sulfonylthiourea 9. Yield, 78\%, white crystalline powder, mp 180-182 ${ }^{\circ} \mathrm{C} .{ }^{1} \mathrm{H}$ NMR (400 MHz, DMSO- $\left.d_{6}\right) \delta$ (ppm). ${ }^{1} \mathrm{H}$ NMR (400 MHz, DMSO- $\left.d_{6}\right) \delta$ (ppm): 9.79 (brs, 2H), 8.72 (brs, $\left.2 \mathrm{H}\right), 7$. 97-7.64 (m, 14H), $7.01(\mathrm{dd}, J=7.3 \mathrm{~Hz}, 1 \mathrm{H}), 6.85(\mathrm{~d}, J=7.3 \mathrm{~Hz}, 2 \mathrm{H}), 6.82(\mathrm{~d}, J=5.3 \mathrm{~Hz}, 1 \mathrm{H})$, $4.76(\mathrm{~d}, J=5.1 \mathrm{~Hz}, 4 \mathrm{H}) .{ }^{13} \mathrm{C}$ NMR $\left(100 \mathrm{MHz}, \mathrm{DMSO}-d_{6}\right) \delta(\mathrm{ppm}): 184.1,141.9,141.5,134.0$, $131.5,130.9,128.4,128.3,126.7,126.6,126.3,125.0,124.5,51.3 . \mathrm{IR}\left(\mathrm{KBr}, \mathrm{cm}^{-1}\right)$ v: 3376, 3182, 3054, 2972, 2929, 1596, 1541, 1501, 1437, 1396, 1272, 1236, 1084, 763, $522 \mathrm{~cm}^{-1}$. ESI-MS m/z: $635.08\left(\mathrm{M}+\mathrm{H}^{+}\right)$. Anal. calcd for $\mathrm{C}_{30} \mathrm{H}_{26} \mathrm{~N}_{4} \mathrm{O}_{4} \mathrm{~S}_{4}$ : C 56.76, $\mathrm{H} 4.13, \mathrm{~N}$ 8.83; found C 56.74, H 4.15, N 8.81.

Sulfonylthiourea 10. Yield, 75\%, white crystalline powder, mp 260-162 ${ }^{\circ} \mathrm{C} .{ }^{1} \mathrm{H}$ NMR (400 MHz, DMSO- $\left.d_{6}\right) \delta(\mathrm{ppm}) .{ }^{1} \mathrm{H}$ NMR (400 MHz, DMSO- $\left.d_{6}\right) \delta$ (ppm): 11.23 (brs, 2H), 8.86 (brs, 2H), $7.50(\mathrm{~s}, 4 \mathrm{H}), 7.45(\mathrm{~m}, 10 \mathrm{H}), 6.47(\mathrm{~s}, 4 \mathrm{H}), 4.38\left(\mathrm{~d}, J_{a x, ~ e q}=14.6 \mathrm{~Hz}, \mathrm{Hax}, 4 \mathrm{H},\right), 4.41-3.87$ (m, $16 \mathrm{H}), 3.17\left(\mathrm{~d}, J_{a x, e q}=14.6 \mathrm{~Hz}\right.$, Heq, $\left.4 \mathrm{H}\right), 1.62(\mathrm{~s}, J=7.5 \mathrm{~Hz}, 6 \mathrm{H}), 1.39(\mathrm{~s}, 18 \mathrm{H}) .{ }^{13} \mathrm{C} \mathrm{NMR}(100$ MHz, DMSO- $\left.d_{6}\right) \delta$ (ppm): 206.1, 157.5, 152.3, 147.3, 146.3, 137.7, 135.4, 127.8, 127.3, 125.6, $125.3,123.4,123.3,73.9,73.5,72.4,72.2,71.9,36.5,33.3,32.4 ; \mathrm{IR}\left(\mathrm{KBr}, \mathrm{cm}^{-1}\right)$ v: 3283, 3225, 1578, 1521, 1145, 1086, 765, $523 \mathrm{~cm}^{-1}$. ESI-MS $\mathrm{m} / z: 1151.39\left(\mathrm{M}+\mathrm{H}^{+}\right)$. Anal. calcd for $\mathrm{C}_{60} \mathrm{H}_{70} \mathrm{~N}_{4} \mathrm{O}_{11} \mathrm{~S}_{4}$ : C 62.58, H 6.13, N 4.87; found C 62.57, H 6.15, N 4.85.

\section{Measurement of sulfonylthiourea-based properties as anion receptors}

The measurements were performed by ${ }^{1} \mathrm{H}$ NMR titration experiments in $\mathrm{CDCl}_{3}-\mathrm{CD}_{3} \mathrm{CN}$ (v/v=4 : 1) at $298 \mathrm{~K}$ using a constant host concentration of $2.0 \mathrm{mM}$ and a varying guest concentration of 0.1-20 mM. For each $K_{\text {ass }}$ value determination, 5-10 different guest concentrations were taken As a probe, the chemical shift of the $\mathrm{SO}_{2} \mathrm{NH}$ signal was used.

In the presence of $\mathrm{K}^{+}$, anion complexation was measured analogously to the above procedure.

The $K_{\text {ass }}$ values were calculated by the equation below as described in literature ${ }^{18}$.

$$
K_{\text {ass }}=\alpha /\left[\left(1^{-} \alpha\right)\left([\mathrm{G}]^{-} \alpha[\mathrm{H}]\right)\right]
$$

where $\alpha=\left(\delta-\delta_{0}\right) /\left(\delta_{\max }-\delta_{0}\right), \delta_{0}$ is the initial chemical shift (host only), $\delta$ is the chemical shift at each titration point, and $\delta_{\max }$ is the chemical shift when the receptor is entirely bound.

\section{Acknowledgements}

We are thankful for financial support from the PhD Foundation of Xinjiang Production and Construction Corps of China (No. 2012BB014).

\section{References}

1. Kondo, S.; Sato, M. Tetrahedron 2006, 62, 4844. 
2. Gunnlaugsson, T.; Kruger, P. E.; Jensen, P.; Tierney, J.; Ali, H. D. P. and Hussey, G. M. J. Org. Chem. 2005, 70, 10875.

3. Wu, B.; Liu, G. G.; Li, M. Q. Chem. Commun. 2011, 3992.

4. Ning, Z.; Yuan, C.; Tian, H. J. Mater. Chem. 2012, 22, 6032.

5. (a) Zhang, H.; Zhang, Y.; Wu, G.Z.; Zhou, J.; Huang, W.; Hu, X. Bioorg. Med. Chem. Lett. 2009, 19, 1740. (b) Yu, D.; Chen, M.; Li, H.; Dai, C.; Wang, Y.; Wang, W.; Xing, H.; Chen, G.; Xu, X. CN 1895674. 2007. Chem. Abstr. 2007, 146, 212730. (c) Wu, G.; Wang, G.; Zhang, H. CN 1569006. 2005. Chem. Abstr. 2005, 144, 64362. (d) Zhang, H.; Zhou, J.; Huang, W.; Wu, G. CN 1560032. 2005. Chem. Abstr. 2005, 143, 326094.

6. Wirth, K.; Englert, H. C.; Bohn, H.; Goegelein, H.; Heitsch, H.; Gerlach, U. DE 19841534, 2000. Chem. Abstr. 2000, 132, 222350.

7. Masereel, B.; Lambert, D. M.; Dogné, J. M.; Poupaert, J. H.; Delarge, J. Epilepsia 1991, 38, 334.

8. (a) Knieps, S.; Englert, H. C.; Gerlach, U.; Goegelein, H.; Heitsch, H.; Lehr, K. H.; PfeifferMarek, S. CN 101080388. 2006. Chem. Abstr. 2006, 145, 83220. (b) Englert, H. C.; Gerlach, U.; Mania, D.; Linz, W.; Goegelein, H.; Klaus, E.; Crause, P. CN 1155545. 1997. Chem. Abstr. 1997, 127, 108846. (c) Englert, H.; Gerlach, U.; Mania, D.; Goegelein, H.; Kaiser, J. CN 1134415. 1996. Chem. Abstr. 1996, 125, 247413. (d) Englert, H.; Gerlach, U.; Crause, P.; Mania, D.; Goegelein, H.; Kaiser, J. CN 1137519. 1996. Chem. Abstr. 1996, 125, 221369. (e) Enlert, H.; Mania, D.; Hartung, J.; Goegelein, H.; Kaiser, J. CN 1111238A. 1995. Chem. Abstr. 1995, 123, 256743.

9. (a) Schoenowsky, H.; Bauer, K.; Bieringer, H. DE 3234450. 1984. Chem. Abstr. 1984, 101, 54744. (b) Saito, K.; Nakagawa, Y.; Yoshioka, T.; Hosaka, H. JP 02160782. 1990. Chem. Abstr. 1990, 113, 211968.

10. Li, J.; Kou, J.; Luo, X.; Fan, E. Tetrahedron Lett. 2008, 49, 2761.

11. Faidallah, H. M.; Basaif, S. A.; Sharshira, E. M.; A-Ba-Oum, A. E. Phosphorus, Sulfur, and. Silicon and the Related Elements, 2002, 177, 671.

12. (a) Levallet, C.; Lerpiniere, J.; Ko, S. Y. Tetrahedron 1997, 53, 5291. (b) Bowser, A. M.; Madalengoitia, J. S. Org. Lett. 2004, 6, 3409. (c) Kang, I. J.; Wang, L. W.; Hsu, S. J.; Lee, C. C.; Lee, Y. C.; Wu, Y. S.; Hsu, T. A.; Yueh, A.; Chao, Y. S.; Chern, J. H. Bioorg. Med. Chem. Lett. 2009, 19, 4134.

13. (a) Li, J.; Zhang, G.; Zhang, Z.; Fan, E. J. Org. Chem. 2003, 68, 1611. (b) Rostom, S. A. F. Bioorg. Med. Chem. 2006, 14, 6475.

14. (a) Ding, C.-W.; Zhang, M.-J; Ma, N. Chin. Chem. Lett. 2009, 20, 1166. (b) Ding,C.W.;Zhang, M.-J; Geng, L.-J.; Li, R.-M. Akivoc 2010, (xi), 49.

15. Gbidini, E.; Ugozzoli, F.; Ungaro, R. J. Am. Chem. Soc. 1990, 112, 6979.

16. Ulirich, H.; Tucker, B.; Sayigh, A. A. R. Tetrahedron 1966, 22, 1565.

17. Upadhyaya, J. S. J.Indian Chem. Soc. 1982, 59, 808.

18. Horman, I.; Dreux, B. Anal. Chem. 1983, 55, 1219. 